\title{
Interação genótipo $x$ ambiente via modelos de normas de reação para características de crescimento em bovinos Nelore
}

\author{
Diego Pagung Ambrosini(1), Carlos Henrique Mendes Malhado(2), \\ Raimundo Martins Filho ${ }^{(3)}$ e Paulo Luiz Souza Carneiro ${ }^{(2)}$
}

\begin{abstract}
(1)Universidade Estadual do Sudoeste da Bahia (Uesb), Campus Juvino de Oliveira, Praça Primavera, no 40, CEP 45700-000 Itapetinga, BA, Brasil. E-mail: diegopagung@yahoo.com.br (2)Uesb, Departamento de Ciências Biológicas, Rua José Moreira Sobrinho, Bairro Jequiezinho, s/no, CEP 45206-190 Jequié, BA, Brasil. E-mail: plscarneiro@gmail.com, carlosmalhado@gmail.com ${ }^{(3)}$ Universidade Federal do Ceará, Campus de Cariri, Avenida Tenente Raimundo Rocha, s/no, Cidade Universitária, CEP 63040-360 Juazeiro do Norte, CE, Brasil. E-mail: martinsfilho@yahoo.com.br
\end{abstract}

Resumo - O objetivo deste trabalho foi avaliar a sensibilidade ao ambiente em bovinos da raça Nelore por meio de diferentes modelos de normas de reação e estimar o progresso genético no gradiente ambiental. Determinaram-se os parâmetros ganho de peso da desmama ao sobreano (GP345) e o peso ajustado aos 205 dias de idade (P205). Um modelo animal padrão (MA), dois modelos hierárquicos de normas de reação com homocedasticidade de variância residual e dois com heterogeneidade foram utilizados. O modelo hierárquico de normas de reação homocedástico com um passo apresentou o melhor ajuste. Os coeficientes de herdabilidade diretos do ambiente baixo para o ambiente alto, no gradiente ambiental, foram de 0,03 a 0,63 e de 0,13 a 0,62 , respectivamente, para GP345 e P205. As correlações entre o intercepto e a inclinação da norma de reação foram de: 0,93, para GP345 (direto); 0,95, para GP345 (materno); 0,92, para P205 (direto); e 0,82, para P205 (materno). As correlações indicam que animais com alto valor genético tendem a responder positivamente aos melhores ambientes. As tendências genéticas mostraram ganhos para os efeitos diretos, principalmente nos melhores ambientes. Há variação genética quanto à sensibilidade dos animais, nos diferentes ambientes, fato que permite a seleção de animais com genótipos mais adequados para a produção em determinado ambiente.

Termos para indexação: avaliação genética, correlação genética, parâmetros genéticos, plasticidade fenotípica, reprodutores, sensibilidade ambiental.

\section{Genotype $x$ environment interaction via models of reaction norms for growth traits in Nelore cattle}

\begin{abstract}
The objective of this work was to evaluate the sensitivity of Nelore cattle to the environment by different models of reaction norms and to estimate the genetic progress in environmental gradient. The parameters weight gain from weaning to yearling (WG345) and adjusted weight at 205 days of age (W205) were determined. A standard animal model (AM), two reaction norm hierarchical models with residual variance homoscedasticity, and two with heterogeneity were used. The homoscedastic reaction norm hierarchical model with one step showed the best fit. The direct heritability coefficients from the low environment to the high one, in environmental gradient, were 0.03 to 0.63 and 0.13 to 0.62 , respectively, for WG345 and W205. The correlations between the intercept and the slope of the reaction norm were: 0.93 , for WG345 (direct); 0.95 , for WG345 (maternal); 0.92, for W205 (direct); and 0.82, for W205 (maternal). The correlations indicate that animals with high genetic value tend to positively respond to better environments. The genetic trends showed gains for the direct effects, mainly in the best environments. There is genetic variation for sensitivity of animals, in different environments, a fact that allows of the selection of animals of more appropriate genotypes for the production in a particular environment.
\end{abstract}

Index terms: genetic evaluation, genetic correlation, genetic parameters, phenotypic plasticity, reproducers, environmental sensitivity.

\section{Introdução}

Nos últimos anos, têm sido registrados ganhos genéticos pequenos em zebuínos da região Nordeste do Brasil (Barbosa et al., 2013; Amaral et al., 2014).
Os autores explicam o pequeno ganho genético como decorrente de problemas na estrutura genética populacional e da orientação aos produtores quanto ao uso dos touros na fazenda. A indicação de material genético, específico de cada sistema de produção, 
poderá aumentar os ganhos, pois, no Brasil, há uma grande variação de ambientes e sistemas de produção, e as avaliações genéticas não consideram a interação genótipo $\mathrm{x}$ ambiente (IGA). A presença da IGA se caracteriza pela resposta diferenciada dos genótipos às variações das condições ambientais, o que pode ocasionar alteração do ordenamento de desempenho dos genótipos no gradiente ambiental (Falconer \& Mackay, 1996). De acordo com Alencar et al. (2005), esta interação pode provocar alterações das variâncias que compõem os parâmetros genéticos e fenotípicos da população, com consequente mudança nos critérios de seleção, a depender do ambiente.

Em geral, os estudos com IGA têm sido desenvolvidos por meio da correlação da mesma característica em ambientes distintos (Costa et al., 2000; Mattos et al., 2000) e funções de covariância (Kirkpatrick et al., 1990; Gomulkiewicz \& Kirkpatrick, 1992). Esta última é uma forma eficiente de se estudar a IGA, pois utiliza a regressão aleatória linear, em que é descrita a mudança gradual e contínua de cada genótipo, em função do gradiente ambiental, ou seja, a norma de reação do animal às variações gradativas do ambiente. Com esta metodologia, vários autores têm verificado a presença da IGA na raça Nelore, em diferentes regiões do Brasil, entre os quais se podem citar: Santana Jr. et al. (2015), quanto ao ganho de peso pós-desmama, circunferência escrotal e produtividade média anual; Chiaia et al. (2015), quanto ao de ganho de peso e reprodução; Raidan et al. (2015), quanto ao peso final e circunferência escrotal em animais Nelore jovens; e Ribeiro et al. (2015), quanto ao peso à desmana.

Em trabalho anterior, avaliou-se a interação genótipo x ambiente, em modelo de normas de reação, quanto à características de crescimento na raça Nelore Mocho (Ambrosini et al., 2014a, 2014b). Os materiais genéticos empregados no presente estudo e nos anteriores são diferentes. $\mathrm{Na}$ raça Nelore, temos os animais conhecidos simplesmente por Nelore (raça Nelore ou também Nelore Padrão) e os animais da raça Nelore variedade Mocha (Nelore Mocho). Estes dois grupos genéticos compõem os maiores efetivos de zebuínos utilizados na região Nordeste do Brasil, e apresentam diferenças além da presença ou não de chifres, e compondo populações muito diferentes.

Assim, é necessário estudar a IGA para estes grupos, pois os resultados - parâmetros genéticos, ganhos genéticos, comportamento ao longo do gradiente ambiental etc. - e o volume de informações de um estudo de IGA são necessários para estes dois grupos genéticos importantes para a região Nordeste.

Estudos do comportamento dos genótipos quanto às variações ambientais são imprescindíveis para $o$ desenvolvimento da bovinocultura de corte na região Nordeste, principalmente os de características de crescimento, que possuem estreita relação com o peso ao abate. Ressalta-se, também, que as avaliações dos ganhos genéticos ao longo do gradiente ambiental podem trazer informações importantes, para nortear ações futuras quanto ao uso de materiais genéticos mais apropriados para a região Nordeste do Brasil.

O objetivo deste trabalho foi avaliar a sensibilidade ao ambiente em bovinos da raça Nelore por meio de diferentes modelos de normas de reação e estimar o progresso genético no gradiente ambiental.

\section{Material de Métodos}

Registros de 112.989 animais da raça Nelore, nascidos entre 1974 e 2011 no Nordeste do Brasil, cedidos pela Associação Brasileira de Criadores de Zebu (ABCZ) foram utilizados. Para a predição do mérito genético em condições de IGA, foram utilizados modelos de normas de reação e avaliados os parâmetros ganho de peso da desmama ao sobreano (GP345) e o peso ajustado aos 205 dias de idade (P205). Os grupos de contemporâneos (GC) foram formados de maneira a identificar apropriadamente a condição ambiental de criação e a permitir melhor comparação do desempenho dos animais. Os efeitos considerados para a formação dos GC foram: ano e época de nascimento do animal; regime alimentar - extensivo, semiestabulado e estabulado; condição de criação e sexo. As épocas de nascimento foram agrupadas em quatro classes: maio a julho; agosto a outubro; novembro a janeiro; fevereiro a abril.

No banco de dados para a análise, foram mantidas, vacas entre 2,3 e 20 anos, com pelo menos duas progênies, e touros com pelo menos cinco progênies com registros de desempenho quanto às características estudadas. Foram excluídos animais pertencentes a GC desconectados, com registros duplicados, sem registros de peso, GC com menos de cinco observações, e GC cuja média fenotípica estava fora do intervalo - média +/- três desvios-padrão. A análise de conectabilidade dos GC foi realizada por meio do programa AMC 
(Roso \& Schenkel, 2006), tendo-se considerado como restrição a existência de no mínimo 10 laços genéticos, em cada GC, e tendo-se mantido apenas animais conectados em 990 e 4787 GC, para GP345 e P205, respectivamente. A média e o desvio-padrão das características $(\mathrm{kg})$ foi de 117,54 e 28, para GP345, e 168,10 e 32,5, para P205. O arquivo de genealogia foi composto por 10.119 animais para o GP345 e 63.571 para o P205, com intervalo de gerações médio de $8,3 \pm 4,2$ anos.

Inicialmente, o Programa Intergen 1.2 (Cardoso, 2010) foi utilizado para empregar um modelo animal padrão (MA) para os dados, de forma a ignorar a IGA. Assim, este modelo possibilitou a predição dos valores genéticos, obtenção das estimativas dos efeitos médios ambientais com base nos GC, para posteriormente serem utilizados como covariáveis nos modelos de normas reação e, também, serviu de base para a comparação entre os modelos:

$$
\mathrm{y}_{\mathrm{ij}}=\mathrm{x}_{\mathrm{i}}^{\prime} \beta+\mathrm{X}_{\mathrm{j}}+\mathrm{a}_{\mathrm{i}}+\mathrm{m}_{\mathrm{i}}+\mathrm{ep}_{\mathrm{i}}+\varepsilon_{\mathrm{ij}}(1)
$$

em que: $y_{\mathrm{ij}}$ é o registro do animal i no ambiente j; $\beta$ é um vetor de efeitos para covariáveis (linear e quadrático para a idade da vaca); $\mathrm{x}_{\mathrm{i}}{ }^{\prime}$ corresponde ao vector incidência; $X_{j}$ é o efeito ambiental aleatório normalmente distribuído, definido como efeitos de $\mathrm{CG} ; \mathrm{a}_{\mathrm{i}}$ é o efeito genético aditivo do animal $\mathrm{i} ; \mathrm{m}_{\mathrm{i}}$ é efeito genético materno do animal $\mathrm{i}$; ep $_{\mathrm{i}}$ é o efeito de ambiente permanente materno; e $\varepsilon_{\mathrm{ij}}$ é o erro aleatório.

Posteriormente, duas metodologias com modelos hierárquicos de normas de reação (MHNR) foram propostos. Nesses modelos, o valor genético é expresso em função de uma covariável ambiental (Falconer, 1990). A primeira metodologia utiliza as estimativas dos efeitos médios ambientais, com base nas soluções de GC obtidos por meio do MA (1), como covariáveis conhecidas em um modelo de regressão aleatória (Kolmodin et al., 2002), chamado aqui de modelo hierárquico norma de reação em dois passos $\left(\mathrm{MHNR}_{2 \mathrm{P}}\right)$ :

$$
y_{i j}=x_{i}^{\prime} \beta+\phi \widehat{X}_{j}+a_{i}+m_{i}+e p_{i}+b_{a i} \widehat{X}_{j}+b_{m i} \widehat{X}_{j}+\varepsilon_{i j}
$$

em que: $\phi$ é o coeficiente de regressão linear fixo de $y_{i j}$ em $\widehat{\mathrm{x}}_{\mathrm{j}} ; \mathrm{a}_{\mathrm{i}}$ é o valor genético aditivo direto do intercepto ou nível da norma de reação do animal i ; $\mathrm{m}_{\mathrm{i}}$ é o valor genético do intercepto para o efeito materno ou nível da norma de reação do animal i; $\mathrm{ep}_{\mathrm{i}}$ é o efeito de ambiente permanente materno; $b_{\mathrm{ai}}$ e $b_{\mathrm{mi}}$ são os coeficientes de regressão aleatórios ou inclinação das normas de reação direta e materna, respectivamente, do animal i no ambiente, representado por $\widehat{\mathrm{X}}_{\mathrm{j}} ; \widehat{\mathrm{x}}_{\mathrm{j}}$ é o preditor de $\mathrm{X}_{\mathrm{j}}$ obtido em (1); e $\varepsilon_{\mathrm{ij}}$ é o erro aleatório. Os demais termos do modelo estão descritos em (1).

Utilizou-se também uma segunda metodologia, proposta por Su et al. (2006), que, apesar da semelhança com (2) apresenta processo de estimação diferente e simultâneo para as soluções dos $\mathrm{GC}\left(\mathrm{X}_{\mathrm{i}}\right)$ e predição das normas de reação, chamado, então, de modelo hierárquico norma de reação em um passo $\left(\mathrm{MHNR}_{1 \mathrm{P}}\right)$ :

$$
y_{i j}=x_{i}^{\prime} \beta+X_{j}+a_{i}+m_{i}+e p_{i}+b_{a i} X_{j}+b_{m i} X_{j}+\varepsilon_{i j}
$$

Assim, são feitas duas pressuposições diferentes acerca da variância residual: homocedasticidade, para MA, $\mathrm{MHNR}_{1 \mathrm{P}}\left(\mathrm{MHNRHO}_{1 \mathrm{P}}\right)$ e $\mathrm{MHNR}_{2 \mathrm{P}}\left(\mathrm{MHNRHO}_{2 \mathrm{P}}\right)$, com $\mathrm{e}_{\mathrm{i}} \sim \mathrm{N}\left(0, \sigma_{\mathrm{e}}^{2}\right)$; e heterocedasticidade, para os modelos $\operatorname{MHNR}_{2 \mathrm{P}}\left(\mathrm{MHNRHE}_{2 \mathrm{P}}\right)$ e $\mathrm{MHNR}_{1 \mathrm{P}}\left(\mathrm{MHNRHE}_{1 \mathrm{P}}\right)$, com $\mathrm{e}_{\mathrm{i}} \sim \mathrm{N}\left(0, \sigma_{\text {eij }}^{2}\right)$.

Após obtenção dos valores genéticos do MA e nos ambientes baixo, médio e alto do modelo de normas de reação - modelo com melhor ajuste para as características avaliadas -, as tendências genéticas foram obtidas pela regressão linear ponderada da média da variável dependente (valores genéticos) sobre o ano de nascimento, por meio do programa SAS versão 9.2 (SAS Institute, Cary, NC, EUA).

Para obtenção das estimativas de todos os parâmetros, utilizou-se o amostrador de Gibbs, por meio do método de Monte Carlo com as cadeias de Markov (MCMC). Nos modelos heterocedásticos, foi necessária a aplicação de um passo extra (Metropolis-Hastings). Todas as cadeias variaram de 55 mil a 880 mil ciclos. O comprimento dependeu da complexidade dos modelos e da quantidade de informações na matriz de parentesco.

Os seguintes procedimentos foram adotados: a, análise-piloto com 55.000 ciclos, 5.500 de descarte ("burn-in") e salvamento a cada cinco amostras ("thinning"); b, com arquivo resposta "varcompsam" e o pacote do programa R (R Foundation for Statistical Computing, Viena, Áustria) "Bayesian output analysis" (BOA), conforme Smith (2007), aplicou-se o teste de Raftery \& Lewis, (1992), para a determinação de um novo tamanho de cadeia e período de salvamento, tendo-se repetido o passo $b$ até alcançar a convergência. O diagnóstico de convergência foi realizado por meio 
do teste Geweke (1992), para todos os parâmetros e diferentes modelos. Este diagnóstico se baseia em um teste $\mathrm{Z}$ de igualdade de médias do logaritmo da distribuição condicional dos dados, denotados pelas primeiras amostras (10\% iniciais) e da última parte da cadeia de Markov (últimas 50\%).

As inferências para todos os componentes de variância foram feitas pela média a posteriori e, para o cálculo de herdabilidade e valor genético, utilizou-se um intervalo de credibilidade de $99 \%$. As amplitudes das soluções dos $\mathrm{GC}\left(\mathrm{X}_{\mathrm{i}}\right)$, representando os níveis baixo e alto, foram de -84 a $96 \mathrm{~kg}$, para GP345 e -72 a $72 \mathrm{~kg}$, para P205. Respeitado o intervalo de credibilidade, as estimativas de herdabilidade e valores genéticos foram calculadas para o gradiente ambiental, com valores entre -69 a $77 \mathrm{~kg}$ e -48 a $52 \mathrm{~kg}$, para GP345 e P205, respectivamente. Os valores foram ajustados com média zero, assim, o valor zero representa o ambiente médio, e as amplitudes mínima e máxima representam os níveis ambientais de baixo a alto manejo no gradiente ambiental, respectivamente. Estes ambientes (baixo, médio e alto) refletem a expectativa da qualidade do ambiente proporcionado aos animais quanto à melhoria e disponibilização de recursos.

Três critérios foram utilizados para verificar a qualidade de ajuste dos modelos: critério de informação da deviance (DIC); deviance baseada na ordenada preditiva condicional (OPC), e a deviance baseada no fator de Bayes (FB). Estes desvios representam o grau de afastamento do modelo avaliado, em relação a um modelo de ajuste hipotético perfeito, em que os menores valores indicam melhor ajuste.

Para valores de inclinações $\left(b_{j}\right)$ próximos de zero, o valor genético $\left(g_{\mathrm{j}}\right)$ será relativamente constante no gradiente ambiental $\left(\mathrm{X}_{\mathrm{i}}\right)$. Então, estes reprodutores têm um genótipo robusto quanto às mudanças ambientais, enquanto os genótipos plásticos são aqueles que alteram substancialmente o seu desempenho no gradiente ambiental, ou seja, têm magnitude elevada de $b_{j}$ (Falconer, 1990). De acordo com o desvio-padrão das inclinações de cada característica, os genótipos dos animais foram classificados em extremamente robusto $\left(\left|b_{j}\right|<\sigma_{b}\right)$, robusto $\left(\sigma_{b} \leq\left|b_{j}\right|<2 \sigma_{b}\right)$, plástico $\left(2 \sigma_{b} \leq\left|b_{j}\right|\right.$ $\left.<3 \sigma_{\mathrm{b}}\right)$ e extremamente plástico $\left(\left|b_{\mathrm{j}}\right| \geq 3 \sigma_{\mathrm{b}}\right)$.

As consequências da IGA, para a seleção em diferentes ambientes, foram avaliadas comparando-se os reprodutores com maior valor genético pelo modelo animal padrão e o modelo de melhor ajuste (ambientes baixo, médio e alto), por meio das correlações de classificação.

\section{Resultados e Discussão}

Observou-se convergência a 1\%, pelo teste Geweke (1992), para os parâmetros dos modelos das características P205 e para maioria dos componentes do GP345. Os modelos de dois passos não convergiram para o GP345 e P205. De acordo com o DIC, o OPC e a FB, as pontuações para o GP345 foram de: 79.131,00, 66.783,26 e 64.044,04, no MA; 76.072,62, 65.595,78 e 63.519,26, para o $\mathrm{MHNRHO}_{1 \mathrm{P}}$; e 78.162,74, 69.369,75 e 71.665,33, para o MHNRHE 1 p. Para o P205, as pontuações foram de: 48.9415,66, 473.844,92 e 399.328,39, no MA; 844.431,16, 429.575,53 e 394.991,15, no $\mathrm{MHNRHO}_{1 \mathrm{P}}$; e 845.220,83, 494.870,40 e 554.322,41, no MHNRHE , $_{1 \mathrm{P}}$. Como valores menores proporcionam ajustes melhores, em ambas as características, o melhor ajuste foi alcançado no MHNRHO $_{1 \mathrm{P}}$.

Estes resultados estão de acordo com Su et al. (2006), que demonstraram, por meio de simulação de dados, que a estimação conjunta de parâmetros desconhecidos é uma abordagem mais efetiva do que usar efeitos ambientais pré-estimados, em modelo animal, como covariáveis em modelos de norma de reação.

As estimativas médias a posteriori \pm desvio-padrão das correlações entre intercepto e inclinação da norma de reação $\left(\mathrm{r}_{\mathrm{ab}}\right)$ foram de: $0,93 \pm 0,04$, para GP345 direto; $0,95 \pm 0,07$, GP345 materno; 0,92 $\pm 0,03$, P205 efeito direto; e 0,82 $\pm 0,09$, P205 efeito materno. Essas altas correlações evidenciam que animais com maior valor genético médio, ao longo do gradiente ambiental, tendem a mostrar aumento do valor genético em ambientes favoráveis. Estes resultados estão próximos aos obtidos por outros trabalhos sobre normas de reação em bovinos de corte - quanto às características de peso (Pégolo et al., 2009; Ambrosini et al., 2014b) e ganho de peso (Cardoso et al., 2011; Chiaia et al., 2015) -, e em bovinos compostos (Santana Jr et al., 2013).

As estimativas médias a posteriori \pm desvio-padrão da herdabilidade direta, no modelo animal padrão (MA), foram de $0,12 \pm 0,03$, para GP345, e $0,34 \pm 0,02$ para P205 (Figura 1). Com a mesma metodologia, Cardoso \& Tempelman (2012) analisaram o GP345, para a raça Angus, e Santana Jr. et al. (2015), para 
bovinos compostos, e estimaram herdabilidades no modelo animal de 0,11 e 0,27 , respectivamente.

Nos MHNR, as estimativas médias a posteriori, da herdabilidade para o efeito direto, foram superiores às do MA nos níveis médio e alto do gradiente ambiental e variaram de 0,04 a 0,48 e 0,13 a 0,62, para GP345 e P205, respectivamente (Figura 1). Para o efeito materno, as estimativas do $\operatorname{MHNR}(0,04$ a 0,19 e 0,02 a 0,13$)$ foram superiores ao do MA $(0,04$, em ambas características) apenas nos melhores ambientes.

Apesar da baixa magnitude, as estimativas de herdabilidade materna indicam que, na fase em que os bezerros ficam aos cuidados da vaca, ainda há influência da mãe sobre o desempenho do bezerro, fato que justifica a inclusão do efeito materno no modelo, para a busca de maior acurácia na partição das variâncias. Pela mesma abordagem na raça Nelore Mocho, Ambrosini et al. (2014b) encontraram, para o P205, estimativas de herdabilidades direta inferiores $(0,08$ a 0,24$)$ às do presente trabalho, no entanto, para o efeito materno, as estimativas foram próximas $(0,03$ a 0,10$)$.

Em diversos estudos (Mattar et al., 2011; Ambrosini et al., 2014a; Ribeiro et al., 2015), observaram-se herdabilidades inferiores às estimativas de modelo animal padrão nos piores ambientes, mas com aumento em ambientes favoráveis. Provavelmente, este comportamento advém do fato de os indivíduos terem melhores condições de expressar o potencial de seus genótipos nos melhores ambientes. Kolmodin et al. (2004) utilizaram modelos de normas de reação e também observaram aumento das estimativas de herdabilidade nos melhores ambientes, que indicam maior resposta à seleção, especialmente se esta é praticada nos ambientes superiores.

As correlações de classificação foram de médias a altas entre os valores genéticos preditos dos reprodutores, nos diferentes ambientes do $\mathrm{MHNRHO}_{1 \mathrm{P}}$, o que mostra a baixa ocorrência de reclassificação, com correlações de 0,78 a 0,99, para GP345, e de 0,72 a 0,99, para P205 (Figura 2). No entanto, mesmo as correlações medianas podem levar a mudanças importantes na classificação dos reprodutores. Ambrosini et al. (2014a) estudaram Nelore Mocho, e Corrêa et al. (2010), a raça Devon, e relataram correlações similares, em que a maioria das alterações na classificação ocorreu entre o ambiente baixo e alto do gradiente ambiental (Santos et al., 2008).

A correlação entre os ambientes médio e alto foi acima de 0,90 para todas as características. Assim, os animais selecionados com base em informações de suas progênies em ambiente médio podem ser utilizados em ambiente alto e vice-versa. Entretanto, a linha do gráfico com as correlações do ambiente baixo com todos os outros ambientes evidenciou correlações menores, o que indica que avaliações genéticas, sem se considerar a IGA, podem levar à equivocada utilização de reprodutores. Isso ocorre principalmente se forem utilizados reprodutores cujas avaliações do mérito genético, no ambiente baixo, mostrem baixo valor genético nos ambientes médio e alto.

As normas de reação dos 10 reprodutores com maior número de filhos reforçam a presença de IGA, com efeito escala, e IGA complexa, principalmente para

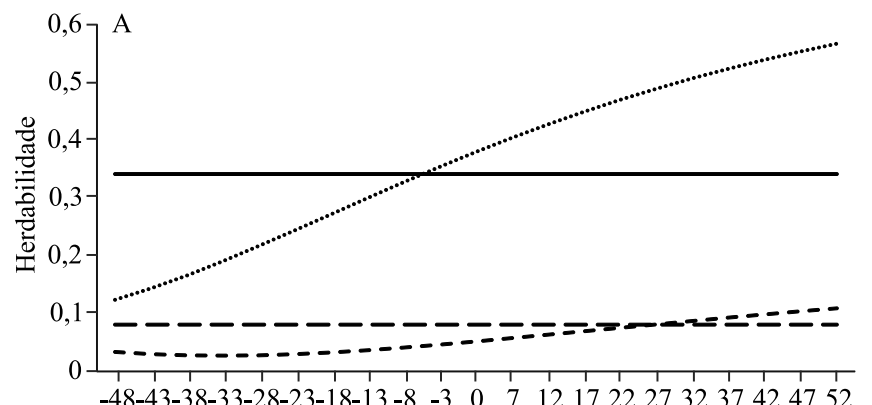

Gradiente ambiental

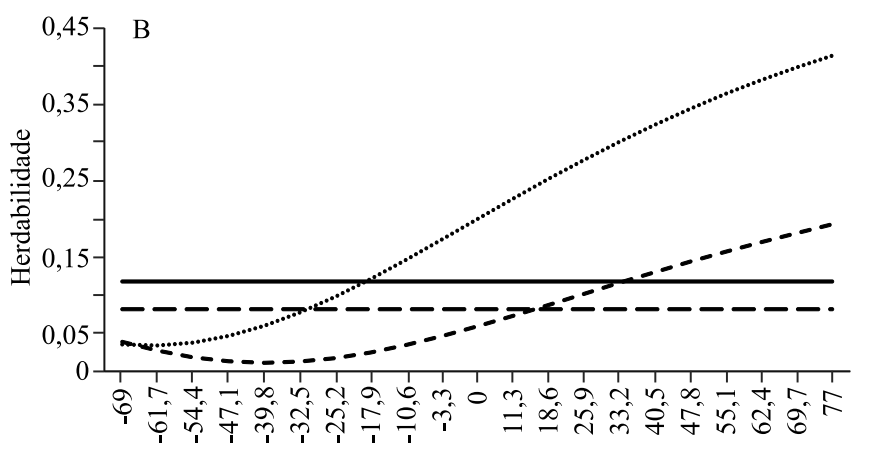

Gradiente ambiental

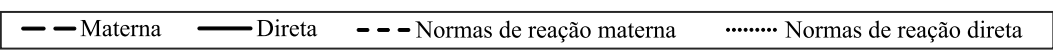

Figura 1. Herdabilidade quanto às características ganho de peso da desmama ao sobreano, GP345 (A) e (B) peso ajustado aos 205 dias de idade, P205, com os efeitos direto e materno, nos modelos propostos no gradiente ambiental. 
os efeitos diretos (Figura 3). No GP345, as retas se cruzam, em sua maioria, nos níveis baixos, contudo, ocorrem ao longo de todo gradiente ambiental, com os valores genéticos dos reprodutores diferenciandose nos ambientes superiores (Figura 3 A). O P205 apresentou comportamento semelhante ao GP345, em que apenas um reprodutor não respondeu positivamente à melhoria ambiental (Figura $3 \mathrm{C}$ ). Apesar da menor variação dos valores genéticos, também foi possível identificar esses efeitos sobre o efeito materno, com as retas se cruzando, em sua maioria, no ambiente baixo. (Figura 3 B e D).

O reprodutor 190 deve ser destacado, uma vez que tem o maior número de filhos para o P205, com coeficiente de inclinação de $-0,23$ (genótipo plástico). Por ser um reprodutor com coeficiente de inclinação negativo, ele responde negativamente à melhoria do gradiente ambiental e apresenta o pior valor genético entre os reprodutores com maior número de filhos. Entretanto, o reprodutor 184 é o segundo em número de filhos para o P205, com coeficiente próximo a 0,21 (genótipo plástico). É um animal superior e está, corretamente, sendo utilizado com maior intensidade.

De acordo com Falconer (1990), a variação da inclinação da norma de reação está diretamente relacionada à IGA e reflete a sensibilidade ambiental dos genótipos na resposta ao ambiente. $\mathrm{O}$ desvio-padrão das inclinações $\left(b_{j}\right)$ foi de: 0,068 ,

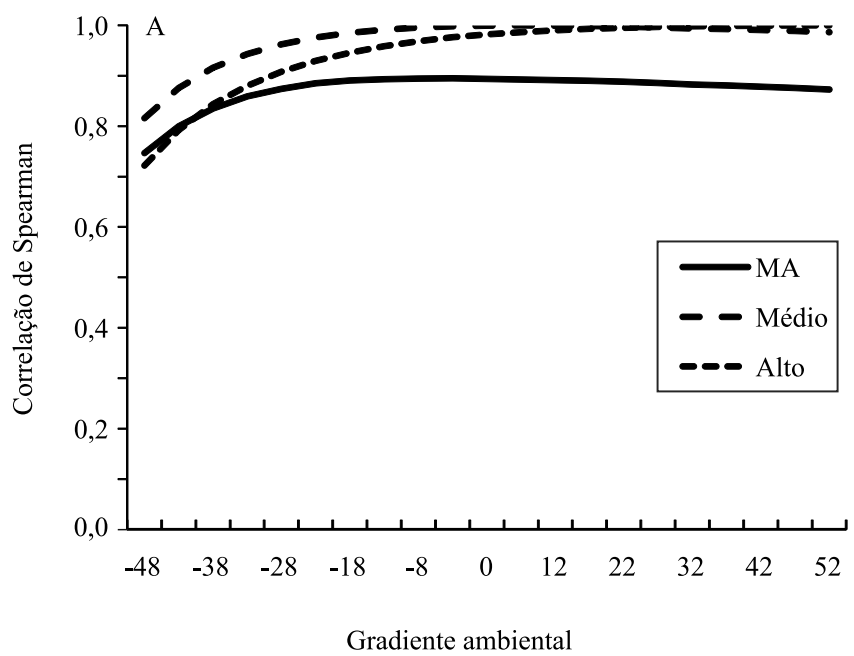

GP345 direto; 0,016, GP345 materno; 0,098, P205 direto; e 0,012, P205 materno. Com base nestes desvios-padrão, a classificação das inclinações dos animais nas classes propostas permitiu observar que, em ambas as características e efeitos, os genótipos são: aproximadamente $75 \%$, extremamente robustos; $20 \%$, robustos, $4 \%$, plásticos; e, apenas $1 \%$, extremamente plásticos. Entretanto, em ambas as características, a média de filhos por reprodutor foi maior nos genótipos plásticos $(45,85$ e 37,80$)$ e extremamente plásticos $(80,06$ e 98,94$)$ do que nos genótipos extremamente robustos $(18,71$ e 34,31$)$ e robustos $(26,82$ e 61,85$)$. Evidencia-se que os genótipos plásticos foram mais utilizados, o que realça a importância de considerar a inclusão da IGA nas avaliações genéticas.

O sistema de produção ideal seria aquele em que os genótipos apresentassem alto desempenho com inclinação próxima a zero, de acordo com Strandberg et al. (2000). Na realidade, um animal não conseguirá produzir sem um mínimo de condições necessárias, não importa quão robusto seja esse genótipo. Vários estudos com normas de reação com Nelore Mocho, Devon, Canchim e Angus (Corrêa et al., 2010; Mattar et al., 2011; Cardoso \& Tempelman, 2012; Ambrosini et al., 2014a, 2014b; e) têm mostrado que animais robustos tendem a ser menos produtivos e a apresentar baixa amplitude de valores genéticos no gradiente ambiental.

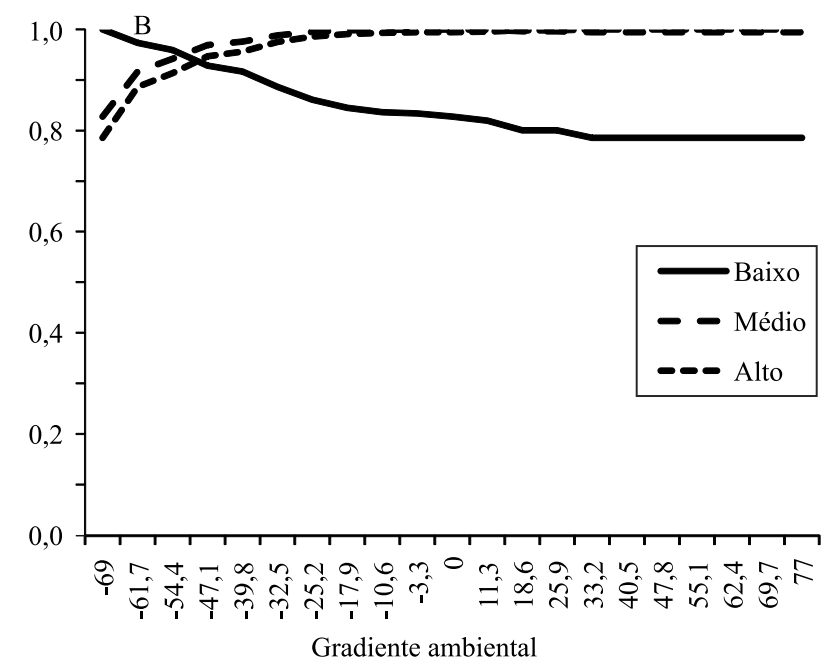

Figura 2. Correlações de Spearman entre os valores genéticos dos reprodutores: A, quanto ao ganho de peso da desmama ao sobreano, GP345; e B, peso ajustado aos 205 dias de idade, P205. As linhas indicam as correlações entre os ambientes baixo (-69 e -48, para GP345 e P205, respectivamente), médio (0) e alto (77 e 47, para GP345 e P205, respectivamente), e todos os outros ambientes. 
Como forma de entender melhor o efeito da IGA sobre os ganhos genéticos desta população, as tendências genéticas para as características foram ajustadas para o MA e para os níveis baixo, médio e alto dos $\mathrm{MHNRHO}_{1 \mathrm{p}}$. Nos efeitos maternos do GP345 e P205, os coeficientes de regressão não foram significativos ( $>00,5)$. Para o GP345 (direto), o ambiente baixo do $\mathrm{MHNRHO}_{1 \mathrm{P}}$ apresentou coeficientes de regressão não significativos $(p>0,489)$, enquanto o MA e o MHNRHO ${ }_{1 \mathrm{P}}$, nos ambientes médio e alto, foram significativos $(p<0,05)$ (Figura 4 A). Os ganhos nos ambientes médio e alto foram muitos pequenos $(0,0430$ e $0,0925 \mathrm{~kg}$ por ano, respectivamente), e o ganho no MA foi inferior a estes $(0,0202 \mathrm{~kg}$ por ano). Estes ganhos são pequenos se comparados com os obtidos por Laureano et al. (2011) que, ao utilizar dados de um programa de melhoramento da mesma raça no Estado de São Paulo, em um modelo animal padrão, encontraram valores bem superiores $(0,224 \mathrm{~kg}$ por ano). Porém, Holanda et al. (2004) relataram mudanças genéticas anuais intermediárias $(0,075 \mathrm{~kg}$ por ano), para a mesma característica na raça Nelore, no Estado de Pernambuco. Ainda, segundo estes autores, apesar de os ganhos genéticos parecerem pequenos, o progresso durante os anos deve ser considerado, pois as tendências são estáveis e cumulativas ao longo dos anos.
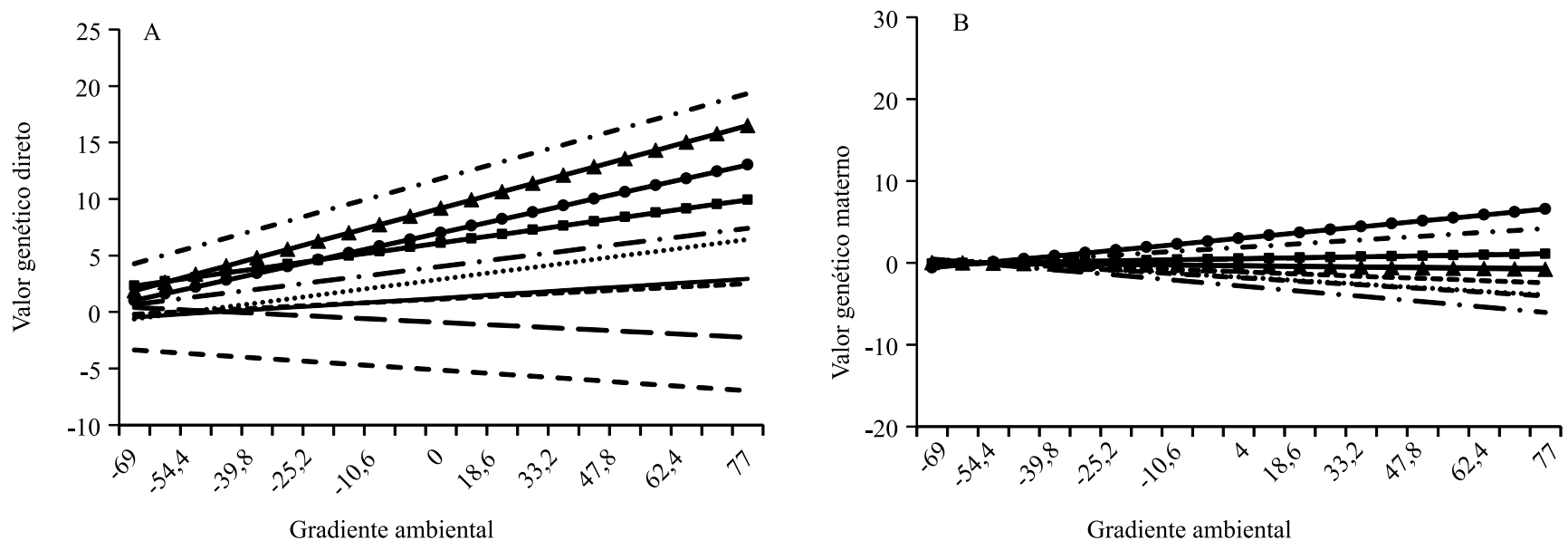

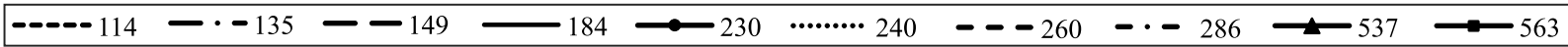

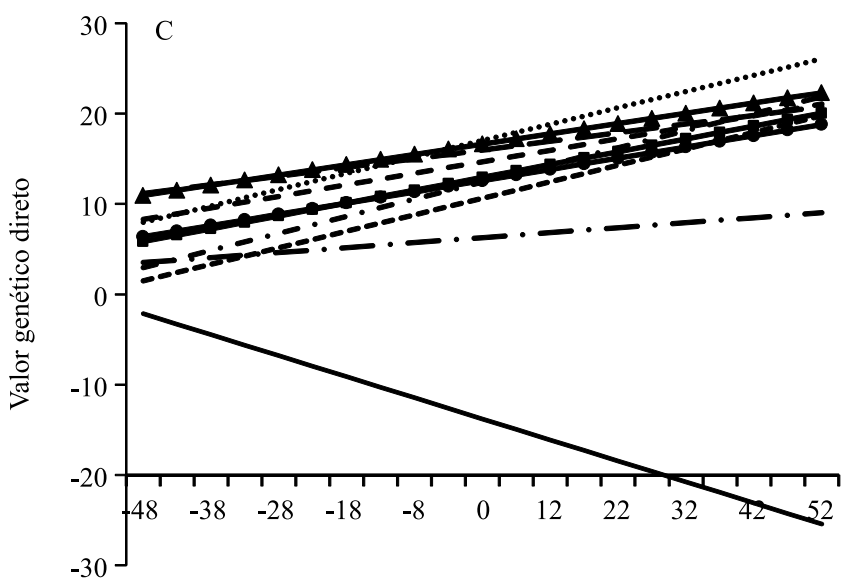

Gradiente ambiental

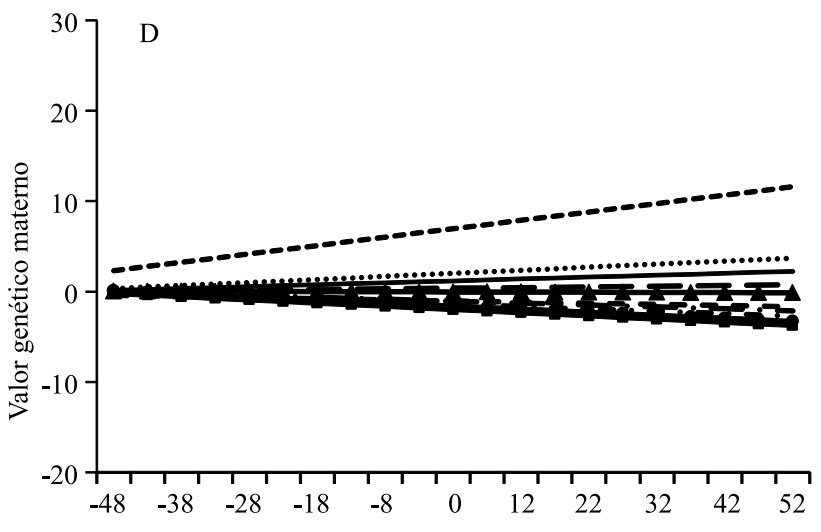

Gradiente ambiental

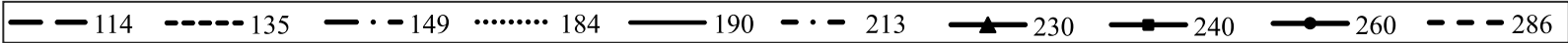

Figura 3. Normas de reação ao longo do gradiente ambiental para as características (A) ganho de peso da desmama ao sobreano, GP345 direto, e (B) peso ajustado aos 205 dias de idade, P205, (C) efeito direto, e (D) efeito materno, obtidos para os 10 reprodutores com maior número de filhos. 

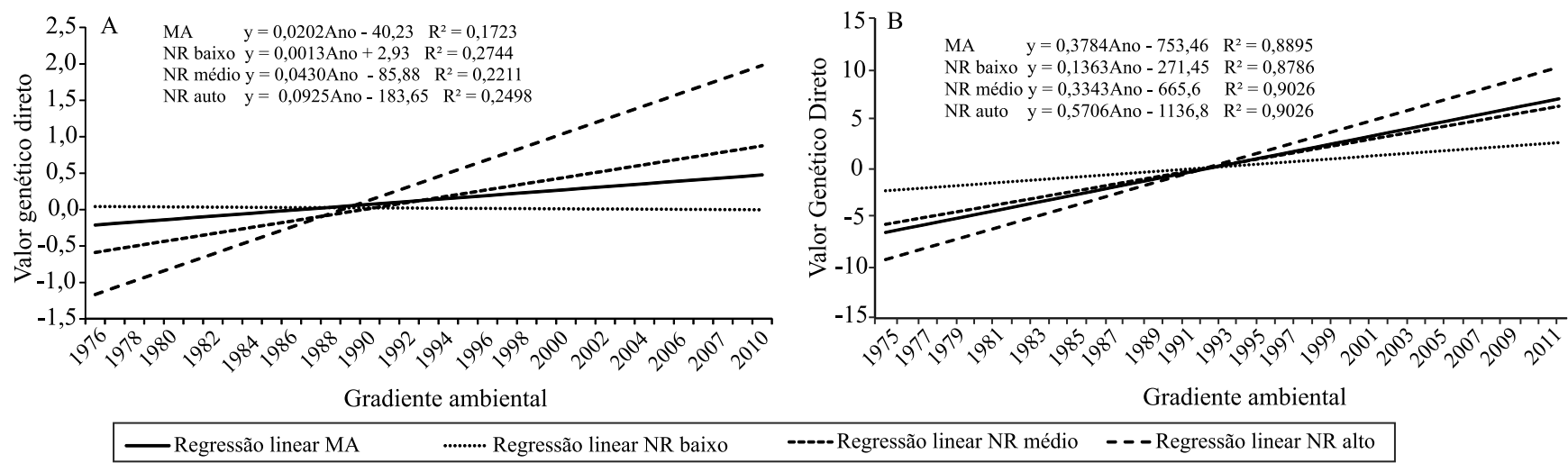

Figura 4. Tendência genética para as características (A) ganho de peso da desmama ao sobreano, GP345, e (B) peso ajustado aos 205 de idade, P205, no modelo animal, e níveis ambientais baixo, médio e alto do modelo de normas de reação, no período de 1976 a 2011.

No P205 (efeito direto), todos os coeficientes de regressão foram significativos $(\mathrm{p}<0,001)$ e positivos $(0,3784,0,1363,0,3343$ e $0,5706 \mathrm{~kg}$ por ano, respectivamente) (Figura $4 \mathrm{~B}$ ). Pelo MA, isto representa o incremento de $13,82 \mathrm{~kg}$, nos 36 anos analisados. Lira et al. (2013), utilizaram dados da mesma raça, nas regiões de trópico úmido do Brasil, e também não encontraram coeficiente de regressão linear significativo para o efeito materno, aos 205 dias de idade, e a tendência genética para o efeito direto, encontrada por estes autores, foi próxima à encontrada no presente trabalho, no MA e ambiente médio $(0,3584 \mathrm{~kg}$ por ano).

A grande vantagem dos MHNR é a seleção de animais para ambientes específicos. Assim, mesmo para ambientes de baixo nível de manejo, em que as diferenças dos valores genéticos dos touros são pequenas, é melhor usar um touro específico, em razão das baixas correlações de classificação, entre os valores genéticos preditos do nível baixo e dos níveis médio e alto. Para os efeitos diretos, em ambas as características, as tendências apresentaram valores similares, crescentes e constantes durante os 36 anos de estudo, o que indica que a seleção de animais para ambientes específicos pode gerar maiores ganhos, desde que seja feita a correta indicação de reprodutores.

Os dados originais desta população Nelore são baseados em avaliações genéticas e seleção de animais, por um modelo animal padrão, ou seja, as análises ignoraram a IGA. Se fossem implementados modelos que contemplassem a inclusão da IGA, durante os 36 anos que compreendem os dados deste estudo, o ganho genético poderia ser maior, já que a seleção e indicação de reprodutores estariam sendo efetivamente aplicadas nos ambientes específicos, com maiores ganhos genéticos.

\section{Conclusões}

1. Os modelos hierárquicos de normas de reação permitem identificar o tipo e a intensidade do efeito da interação genótipo por ambiente sobre os animais e podem ser utilizados para maximizar o progresso genético, em condições variáveis de ambiente.

2. As estimativas dos valores genéticos e das herdabilidades aumentam com a melhoria ambiental e mostram possibilidades de ganhos genéticos na seleção e uso de genótipos, em ambientes de médio e alto manejo.

3. Pode-se obter progresso genético para o desempenho do bovino Nelore, principalmente nos melhores ambientes.

\section{Agradecimentos}

Ao Conselho Nacional de Desenvolvimento Científico e Tecnológico (CNPq), pelas bolsas de produtividade concedidas a Carlos Henrique Mendes Malhado e a Paulo Luiz Souza Carneiro; à Fundação de Amparo à Pesquisa do Estado da Bahia (Fapesb), pelo apoio financeiro; à Universidade Estadual do Sudoeste da Bahia (Uesb); e à Associação Brasileira de Criadores de Zebu (ABCZ), por ceder os dados. 


\section{Referências}

ALENCAR, M.M. de; MASCIOLI, A. dos S.; FREITAS, A.R. de. Evidências de interação genótipo $\mathrm{x}$ ambiente sobre características de crescimento em bovinos de corte. Revista Brasileira de Zootecnia, v.34, p.489-495, 2005. DOI: 10.1590/S1516-35982005000200016.

AMARAL, R. dos S.; CARNEIRO, P.L.S.; MARTINS FILHO, R.; AMBROSINI, D.P.; MALHADO, C.H.M.Tendências, parâmetros fenotípicos e genéticos de características de crescimento em bovinos Nelore mocho do Nordeste brasileiro. Revista Brasileira de Saúde e Produção Animal, v.15, p.261-271, 2014. DOI: 10.1590/S1519-99402014000200003.

AMBROSINI, D.P.; CARNEIRO, P.L.S.; BRACCINI NETO, J.; MARTINS FILHO, R.; AMARAL, R.D.S.; CARDOSO, F.F.; MALHADO, C.H.M. Reaction norms models in the adjusted weight at 550 days of age for Polled Nellore cattle in Northeast Brazil. Revista Brasileira de Zootecnia, v.43, p.351-357, 2014a. DOI: 10.1590/S1516-35982014000700002.

AMBROSINI, D.P.; MALHADO, C.H.M.; BRACCINI NETO, J.; MARTINS FILHO, R.; AFFONSO, P.R.A. de M.; CARNEIRO, P.L.S. Reaction norms of direct and maternal effects for weight at 205 days in Polled Nellore cattle in north-eastern Brazil. Archiv Tierzucht, v.57, p.1-11, 2014b. DOI: 10.7482/0003-9438-57-032.

BARBOSA, A.C.B.; MALHADO, C.H.M.; CARNEIRO, P.L.S.; MUNIZ, L.M.S.; AMBROSINI, D.P.; CARRILLO, J.A.; MARTINS FILHO, R. Population structure of Nellore cattle in northeastern Brazil. Revista Brasileira de Zootecnia, v.42, p.639-644, 2013. DOI: 10.1590/S1516-35982013000900005.

CARDOSO, F.F. Aplicação da inferência bayesiana no melhoramento animal usando o programa Intergen: manual da versão 1.2. Bagé: Embrapa Pecuária Sul, 2010. 31p. (Embrapa Pecuária Sul. Documentos, 111).

CARDOSO, F.F.; TEMPELMAN, R.J. Linear reaction norm models for genetic merit prediction of Angus cattle under genotype by environment interaction. Journal of Animal Science, v.90, p.2130-2141, 2012. DOI: 10.2527/jas.2011-4333.

CARDOSO, L.L.; BRACCINI NETO, J.; CARDOSO, F.F.; COBUCI, J.A.; BIASSUS, I. de O.; BARCELLOS, J.O.J. Hierarchical Bayesian models for genotype $\times$ environment estimates in post-weaning gain of Hereford bovine via reaction norms. Revista Brasileira de Zootecnia, v.40, p.294-300, 2011. DOI: $10.1590 /$ S1516-35982011000200009.

CHIAIA, H.L.J.; LEMOS, M.V.A. de; VENTURINI, G.C.; ABOUJAOUDE, C.; BERTON, M.P.; FEITOSA, F.B.; CARVALHEIRO, R.; ALBUQUERQUE, L.G.; OLIVEIRA, H.N. de; BALDI, F. Genotype $\times$ environment interaction for age at first calving, scrotal circumference, and yearling weight in Nellore cattle using reaction norms in multitrait random regression models. Journal of Animal Science, v.93, p.1503-1510, 2015. DOI: 10.2527/jas.2014-8217.

CORRÊA, M.B.B.; DIONELLO, N.J.L.; CARDOSO, F.F. Genetic evaluation of Devon Cattle using a reaction norms model. Revista Brasileira de Zootecnia, v.39, p.128-133, 2010. DOI: 10.1590/ S1516-35982010000100017.
COSTA, C.N.; BLAKE, R.W.; POLLAK, E.J.; OLTENACU, P.A.; QUAAS, R.L.; SEARLE, S.R. Genetic analysis of Holstein cattle populations in Brazil and the United States. Journal of Dairy Science, v.83, p.2963-2974, 2000. DOI: $10.3168 /$ jds. S0022-0302(00)75196-4.

FALCONER, D.S. Selection in different environments: effects on environmental sensitivity (reaction norm) and on mean performance. Genetical Research, v.56, p.57-70, 1990. DOI: 10.1017/S0016672300028883.

FALCONER, D.S.; MACKAY, T.F.C. Introduction to quantitative genetics. $4^{\text {th }}$ ed. Essex: Longman: Pearson, 1996. 464p.

GEWEKE, J. Evaluating the accuracy of sampling-based approaches to the calculation of posterior moments. In: BERNARDO, J.M.; BERGER, J.O.; DAWID, A.P.; SMITH, A.F.M. (Ed.). Bayesian statistics 4: proceedings of the Fourth Valencia International Meeting, April 15-20, 1991. New York: Oxford University Press, 1992. p.169-93.

GOMULKIEWICZ, R.; KIRKPATRICK, M. Quantitative genetics and the evolution of reaction norms. Evolution, v.46, p.390-411, 1992. DOI: $10.2307 / 2409860$.

HOLANDA, M.C.R.; BARBOSA, S.B.P.; RIBEIRO, A.C.; SANTORO, K.R. Tendências genéticas para crescimento em bovinos Nelore em Pernambuco, Brasil. Archivos de Zootecnia, v.53, p.185-194, 2004.

KIRKPATRICK, M.; LOFSVOLD, D.; BULMER, M. Analysis of the inheritance, selection and evolution of growth trajectories. Genetics, v.124, p.979-993, 1990.

KOLMODIN, R.; STRANDBERG, E.; DANELL, B.; JORJANI, $\mathrm{H}$. Reaction norms for protein yield and days open in Swedish red and white dairy cattle in relation to various environmental variables. Acta Agriculturae Scandinavica, Section A - Animal Science, v.54, p.139-151, 2004. DOI: 10.1080/09064700410032040.

KOLMODIN, R.; STRANDBERG, E.; MADSEN, P.; JENSEN, J.; JORJANI, H. Genotype by environment interaction in Nordic dairy cattle studied using reaction norms. Acta Agriculturae Scandinavica, Section A - Animal Science, v.52, p.11-24, 2002. DOI: $10.1080 / 09064700252806380$.

LAUREANO, M.M.M.; BOLIGON, A.A.; COSTA, R.B.; FORNI, S.; SEVERO, J.L.P.; ALBUQUERQUE, L.G. Estimativas de herdabilidade e tendências genéticas para características de crescimento e reprodutivas em bovinos da raça Nelore. Arquivo Brasileiro de Medicina Veterinária e Zootecnia, v.63, p.143-152, 2011. DOI: 10.1590/S0102-09352011000100022.

LIRA, T.S. de; PEREIRA, L. de S.; LOPES, F.B.; FERREIRA, J.L.; LÔBO, R.B.; SANTOS, G.C. de J. Tendências genéticas para características de crescimento em rebanhos Nelore criados na região do trópico úmido do Brasil. Ciência Animal Brasileira, v.14, p.23-31, 2013. DOI: 10.5216/cab.v14i1.16785.

MATTAR, M.; SILVA, L.O.C.; ALENCAR, M.M.; CARDOSO, F.F. Genotype $\times$ environment interaction for long-yearling weight in Canchim cattle quantified by reaction norm analysis. Journal of Animal Science, v.89, p.2349-2355, 2011. DOI: 10.2527/ jas.2010-3770. 
MATTOS, D. de; BERTRAND, J.K.; MISZTAL, I. Investigation of genotype $\mathrm{x}$ environment interactions for weaning weight for Herefords in three countries. Journal of Animal Science, v.78, p.2121-2126, 2000.

PÉGOLO, N.T.; OLIVEIRA, H.N. de; ALBUQUERQUE, L.G.; BEZERRA, L.A.F.; LÔBO, R.B. Genotype by environment interaction for 450-day weight of Nelore cattle analyzed by reaction norm models. Genetics and Molecular Biology, v.32, p.281-287, 2009. DOI: $10.1590 / \mathrm{S} 1415-47572009005000027$.

RAFTERY, A.E.; LEWIS, S.M. Comment: one long run with diagnostics: implementation strategies for Markov chain Monte Carlo. Statistical Science, v.7, p.493-497, 1992. DOI: 10.1214/ ss/1177011143.

RAIDAN, F.S.S.; PASSAFARO, T.L.; FRAGOMENI, B.O.; JOSAHKIAN, L.A.; PEREIRA, I.G.; TORAL, F.L.B. Genotype $\times$ environment interaction in individual performance and progeny tests in beef cattle. Journal of Animal Science, v.93, p.920-933, 2015. DOI: $10.2527 /$ jas.2014-7983.

RIBEIRO, S.; ELER, J.P.; PEDROSA, V.B.; ROSA, G.J.M.; FERRAZ, J.B.S.; BALIEIRO, J.C.C. Genotype $\times$ environment interaction for weaning weight in Nellore cattle using reaction norm analysis. Livestock Science, v.176, p.40-46, 2015. DOI: 10.1016/j.livsci.2015.03.032.

ROSO, V.M.; SCHENKEL, F.S. AMC: a computer program to assess the degree of connectedness among contemporary groups. In: WORLD CONGRESSON GENETICS APPLIED TO LIVESTOCK PRODUCTION, 8., 2006, Belo Horizonte. Proceedings. Belo Horizonte: Instituto Prociência, 2006. p.26-27.
SANTANA JR, M.L.; ELER, J.P.; CARDOSO, F.F.; ALBUQUERQUE, L.G.; FERRAZ, J.B.S. Phenotypic plasticity of composite beef cattle performance using reaction norms model with unknown covariate. Animal, v.7, p.202-210, 2013. DOI: $10.1017 /$ S 1751731112001711 .

SANTANA JR., M.L.; ELER, J.P.; BIGNARDI, A.B.; MENÉNDEZ-BUXADERA, A.; CARDOSO, F.F.; FERRAZ, J.B.S. Multi-trait linear reaction norm model to describe the pattern of phenotypic expression of some economic traits in beef cattle across a range of environments. Journal of Applied Genetics, v.56, p.219-229, 2015. DOI: 10.1007/s13353-014-0242-9.

SANTOS, G.G.; CORRÊA, G.S.S.; VALENTE, B.D.; SILVA, M.A.; CORRÊA, A.B.; FELIPE, V.P.S.; WENCESLAU, R.R. Sensibilidade dos valores genéticos de codornas de corte em crescimento às modificações de níveis de proteína das dietas. Arquivo Brasileiro de Medicina Veterinária e Zootecnia, v.60, p.1188-1196, 2008. DOI: 10.1590/S0102-09352008000500022.

SMITH, B.J. Boa: an R Package for MCMC output convergence. Journal of Statistical Software, v.21, p.1-37, 2007. DOI: 10.18637/jss.v021.i11.

STRANDBERG, E.; KOLMODIN, R.; MADSEN, P.; JENSEN, J.; JORJANI, H. Genotype by environment interaction in Nordic dairy cattle studied by use of reaction norms. Interbull Bulletin, v. 25, p. $41-45,2000$.

SU, G.; MADSEN, P.; LUND, M.S.; SORENSEN, D.; KORSGAARD, I.R.; JENSEN, J. Bayesian analysis of the linear reaction norm model with unknown covariates. Journal of Animal Science, v.84, p.1651-1657, 2006. DOI: 10.2527/jas.2005-517.

Recebido em 17 de julho de 2015 e aprovado em 6 de novembro de 2015

Pesq. agropec. bras., Brasília, v.51, n.2, p.177-186, fev. 2016

DOI: $10.1590 / \mathrm{S} 0100-204 \mathrm{X} 2016000200010$ 